\title{
Clinical Profile and Visual Outcome of Ocular Bartonellosis in Malaysia
}

\author{
Chai Lee Tan, ${ }^{1}$ Lai Chan Fhun, ${ }^{1,2}$ Evelyn Li Min Tai, ${ }^{1}$ \\ Nor Hasnida Abdul Gani, ${ }^{3}$ Julieana Muhammed, ${ }^{1}$ Tengku Norina Tuan Jaafar, ${ }^{3}$ \\ Liza Sharmini Ahmad Tajudin, ${ }^{1}$ and Wan-Hazabbah Wan Hitam ${ }^{1}$ \\ ${ }^{1}$ Department of Ophthalmology, School of Medical Sciences, Universiti Sains Malaysia, Health Campus, \\ 16150 Kubang Kerian, Kelantan, Malaysia \\ ${ }^{2}$ Department of Ophthalmology, Hospital Raja Permaisuri Bainun, 30990 Ipoh, Perak, Malaysia \\ ${ }^{3}$ Department of Ophthalmology, Hospital Raja Perempuan Zainab 2, 15586 Kota Bharu, Kelantan, Malaysia \\ Correspondence should be addressed to Evelyn Li Min Tai; daileid@yahoo.com
}

Received 29 August 2016; Revised 20 December 2016; Accepted 10 January 2017; Published 7 February 2017

Academic Editor: Marcel Tanner

Copyright (c) 2017 Chai Lee Tan et al. This is an open access article distributed under the Creative Commons Attribution License, which permits unrestricted use, distribution, and reproduction in any medium, provided the original work is properly cited.

\begin{abstract}
Background. Ocular bartonellosis can present in various ways, with variable visual outcome. There is limited data on ocular bartonellosis in Malaysia. Objective. We aim to describe the clinical presentation and visual outcome of ocular bartonellosis in Malaysia. Materials and Methods. This was a retrospective review of patients treated for ocular bartonellosis in two ophthalmology centers in Malaysia between January 2013 and December 2015. The diagnosis was based on clinical features, supported by a positive Bartonella spp. serology. Results. Of the 19 patients in our series, females were predominant (63.2\%). The mean age was 29.3 years. The majority $(63.2 \%)$ had unilateral involvement. Five patients $(26.3 \%)$ had a history of contact with cats. Neuroretinitis was the most common presentation (62.5\%). Azithromycin was the antibiotic of choice (42.1\%). Concurrent systemic corticosteroids were used in approximately $60 \%$ of cases. The presenting visual acuity was worse than $6 / 18$ in approximately $60 \%$ of eyes; on final review, $76.9 \%$ of eyes had a visual acuity better than 6/18. Conclusion. Ocular bartonellosis tends to present with neuroretinitis. Azithromycin is a viable option for treatment. Systemic corticosteroids may be considered in those with poor visual acuity on presentation.
\end{abstract}

\section{Introduction}

Cat-scratch disease is caused by the Gram-negative, intracellular bacteria Bartonella henselae. When ocular involvement is present, the disease is termed "ocular bartonellosis." The spectrum of ocular bartonellosis manifestation is wide, ranging from neuroretinitis to Parinaud's oculoglandular syndrome, which is an unusual syndrome characterized by granulomas in the eye, preauricular lymphadenopathy, and positive skin test to Bartonella spp. antigens [1-4]. In Malaysia, the data on cat-scratch disease, particularly ocular bartonellosis, is limited [5]. We aim to describe the clinical manifestations and visual outcome of ocular bartonellosis in Malaysia. We also outline the relevant investigations for this condition and discuss the management of this zoonotic disease.

\section{Materials and Methods}

We performed a retrospective review of medical records of patients with ocular bartonellosis treated in two ophthalmology centers in Malaysia, between 2013 and 2015. The conduct of the study followed the tenets outlined in the Declaration of Helsinki.

Selection criteria for this study were a diagnosis of ocular bartonellosis based on history of exposure to cats, ocular examination findings, and a single positive serology for Bartonella spp. Any two of these were sufficient to make the diagnosis. Patients suspected of ocular bartonellosis were also investigated for other possible infective causes (tuberculosis, syphilis). Serological tests were all performed via immunofluorescence assay (IFA) in the Bacteriology Unit, Institute for Medical Research, Ministry of Health 
TABLE 1: Patient demographic profile.

\begin{tabular}{lc}
\hline Demographic data & $n(\%)$ \\
\hline Age in year (mean, SD) & $29.3,13.2$ \\
\hline Race & $19(100.0)$ \\
$\quad$ Malay & $7(36.8)$ \\
\hline Sex & $12(63.2)$ \\
$\quad$ Male & \\
Female & $14(73.7)$ \\
\hline Cat exposure & $5(26.3)$ \\
No & \\
Yes (contact or scratch) & $8(42.1)$ \\
\hline Systemic symptoms & $11(57.9)$ \\
No & $7(43.8)$ \\
Yes & $2(12.5)$ \\
$\quad$ Headache & $6(37.5)$ \\
Upper respiratory tract infection & $1(6.2)$ \\
$\quad$ Fever &
\end{tabular}

Malaysia. The cut off value was 1:12 for $\operatorname{Ig} M$ and 1:64 for Ig $\mathrm{G}$ using a commercial kit to detect Bartonella henselae and Bartonella quintana (Vircell IFA Bartonella, Paraque Tecnologico, Granada, Spain).

Data collected included onset and duration of visual disturbance, associated systemic symptoms, visual acuity, optic nerve function, anterior and posterior segment examination, treatment regime, and visual outcome. The latter was evaluated based on visual acuity at the last follow-up visit. Visual acuity was assessed monocularly, with the patient seated 6 meters from a Snellen chart. A 6/6 Snellen visual acuity means the patient can read at 6 meters a letter that a normal person would be able to read at 6 meters. The denominator in Snellen visual acuity notation describes the distance at which a normal individual is able to identify a certain symbol, while the numerator refers to the distance required by the patient to identify the same symbol. In this study, a visual acuity of $6 / 18$ or better was considered good, while visual acuity worse than $6 / 18$ was considered poor. Follow-up was until completion of the course of antibiotics, given for a minimum of six weeks.

The data were analyzed using the Statistical Package for Social Science, version 22.0 (SPSS Inc., Chicago, IL, USA). The results are presented as frequencies and percentages.

\section{Results}

A total of 19 patients (26 eyes) with ocular bartonellosis were diagnosed within the study period. The period of follow-up ranged from 3 to 68 weeks, with the mean duration of followup being 23.4 weeks. Two patients were lost to follow-up. The duration of symptoms ranged from 5 days to 1 month prior to their first consultation at eye clinic.

The demographic and clinical profile of our subjects is summarized in Table 1 . The mean age was 29.3 years. All were Malay, and most of them were female $(63.2 \%, n=12)$.
Only $5(26.3 \%)$ patients gave a history of being scratched or bitten by a cat. The majority $(63.2 \%, n=12)$ had unilateral involvement.

The most common ocular complaint was blurring of vision, with $61.6 \%$ (16 eyes) presenting with a visual acuity worse than 6/18. About one-quarter (7 eyes) had a visual acuity of worse than $6 / 60$. Approximately $62.5 \%$ of eyes presented with neuroretinitis. Optic disc edema was seen in $42.1 \%$ of patients. One patient presented with features of vasculitis complicated with central retinal vein occlusion, evidenced by macular edema, dilated and tortuous vessels, and flameshaped hemorrhages. Six patients (31.6\%) had signs of uveitis. Ocular findings on presentation and treatment regimes are shown for each patient in Table 2 . Table 3 shows a summary of the ocular presentation and results of serological testing.

Approximately $57.9 \%$ of patients had prodromal symptoms, of which headache was the most common. Less common symptoms were fever and upper respiratory tract symptoms. Bartonella spp. Ig G serology was positive in all patients, while $68.4 \%(n=13)$ had positive Ig M serology. Only a single serology test was performed, due to financial constraints. Hence, disease progression was assessed based on clinical response. The duration of ocular symptoms did not vary between patients with positive Ig $G$ and negative Ig $\mathrm{M}$ and those who were positive for both of these.

All patients except two received treatment, which was initiated within the first week of diagnosis. In the case of the latter, this was due to spontaneous recovery before treatment could be initiated. Azithromycin was the most common antimicrobial agent used for treatment (42.1\%). Doxycycline, ciprofloxacin, ceftazidime, and cotrimoxazole were other agents used. Approximately 57.9\% $(n=11)$ received systemic corticosteroid therapy. Six of these patients were treated with pulsed intravenous corticosteroid therapy, followed by oral corticosteroids. The overall final visual acuity was good, with approximately $76.9 \%$ of eyes achieving a good visual outcome (visual acuity of $6 / 18$ or better).

\section{Discussion}

Cat-scratch disease, that is, bartonellosis, is a self-limiting condition in immunocompetent individuals. The spectrum of systemic involvement in bartonellosis ranges from endocarditis to thyroiditis, arthritis, haemolytic anemia, and glomerulonephritis [6-10]. The ocular manifestations of Bartonella spp. infection are likewise myriad and have included scotomas [11], papillitis, [12] retinal artery occlusions [13], and even a macular hole [14]. Although systemic cat-scratch disease primarily affects young patients, ocular bartonellosis has a broader age distribution [15]. Our mean age of 29.3 years, with a range of 9 to 58 years, is similar to that of Curi et al. in Brazil [2].

Cats are the main reservoir for Bartonella spp., and the main vector of cat-scratch disease is the flea Ctenocephalides felis [16]. Cats are common in Malaysia, both as household pets and strays. The prevalence of Bartonella henselae in fleas from healthy cats and dogs has been shown to be approximately $33 \%$, while among strays, almost a quarter of 


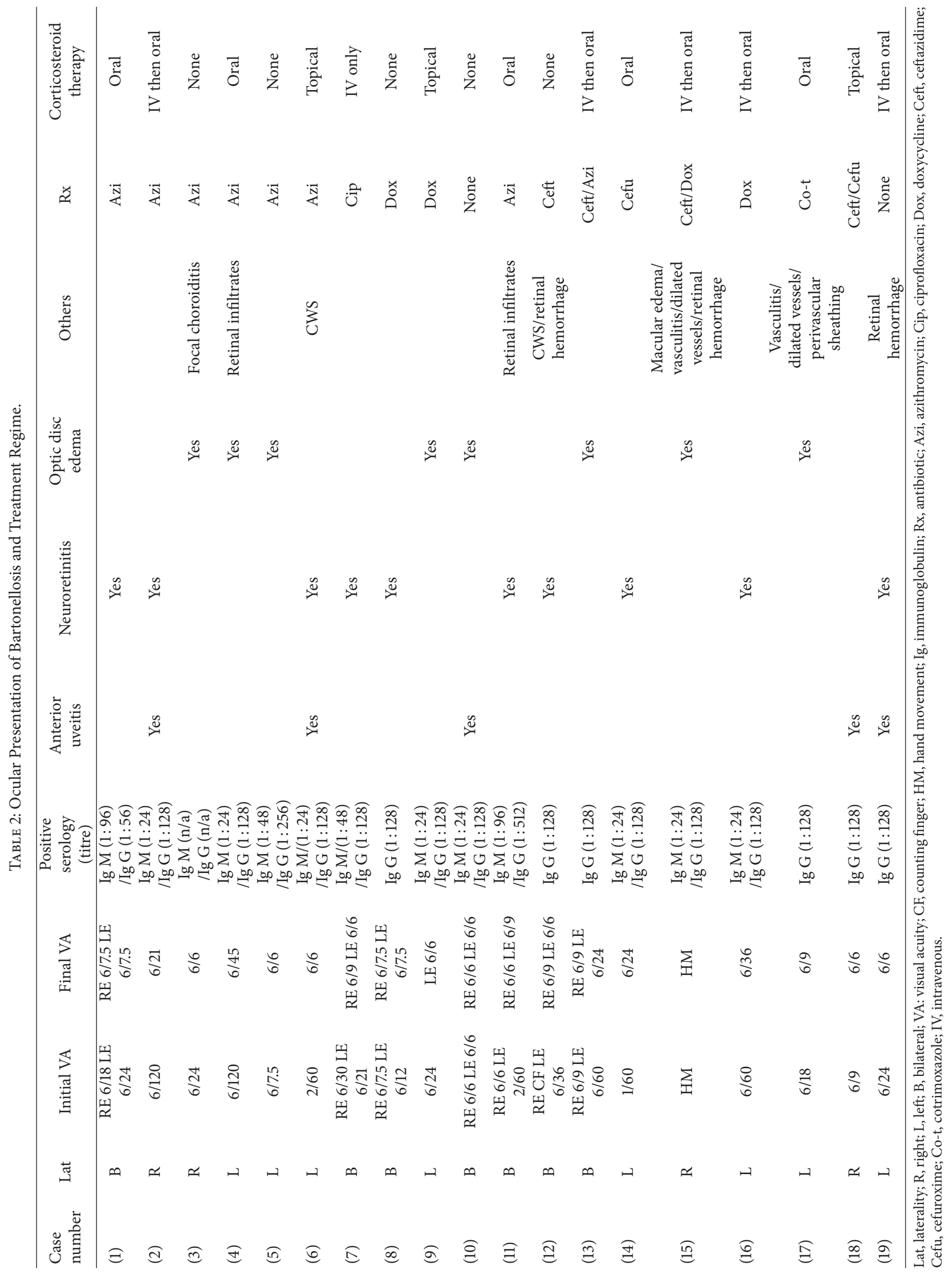


TABle 3: Presenting features of ocular bartonellosis.

\begin{tabular}{lc}
\hline Parameters & $n(\%)$ \\
\hline Laterality & $8(42.1)$ \\
Left & $4(21.1)$ \\
Right & $7(36.8)$ \\
Bilateral & \\
\hline Ocular presentation & $6(31.6)$ \\
Anterior uveitis & $10(62.5)$ \\
Neuroretinitis & $8(42.1)$ \\
Optic disc edema & $1(5.3)$ \\
Focal choroiditis & $2(10.5)$ \\
Retinal infiltrates & $1(5.3)$ \\
Cotton wool spots & $3(15.8)$ \\
Retinal hemorrhages & $1(5.3)$ \\
Macular edema & $2(10.5)$ \\
Vasculitis & $13(68.4)$ \\
\hline IFA serology positivity & $19(100.0)$ \\
Ig M &
\end{tabular}

IFA, immunofluorescent assay; Ig, immunoglobulin.

them have been found to be seropositive for the bacterium $[12,17]$. Exposure to cats or a history of cat-scratch or cat bite is usually present in more than $50 \%$ of reported cases of bartonellosis [2, 18-20]. A recent study among healthy blood donors in Brazil revealed that cat contact or a history of tick bite conferred an adjusted odds ratio of Bartonella spp. bloodstream infection of 3.4 (95\% CI 1.1-9.6) and 3.7 (95\% CI 1.3-13.4), respectively [21]. Our study results contradict those, as only approximately one-quarter of our patients had this history. We thus counsel the reader that although a history of cat exposure is common, it is not a prerequisite to make the diagnosis, especially in regions where cats abound [22].

Neuroretinitis in ocular bartonellosis is usually unilateral, but bilateral presentation has been reported [2, 23-25]. In our study, $63.2 \%$ of patients presented with unilateral involvement. This is similar to the percentage noted by Kerkhoff et al., who noted that $61.5 \%$ of ocular bartonellosis was unilateral [18]. Nearly all (8 out of 9) patients with neuroretinitis had unilateral involvement. Meanwhile, all those with panuveitis (4 patients) had bilateral involvement [18]. Our results differ from those of Curi et al., who reported that 13 out of 24 patients (54.2\%) presented with bilateral disease [2].

Neuroretinitis and focal retinochoroiditis are the most common ocular manifestations of cat-scratch disease $[2,19$, $20,23,24]$. In our series, neuroretinitis remained the most common presentation, followed by optic disc swelling. This varies from the findings of Solley et al., who reported that, in his study of 24 patients, the most common finding was discrete retinal infiltrates or choroidal lesions, seen in $83 \%$ of patients, followed by optic disc swelling, in $63 \%$ of patients [1]. Curi et al. had similar findings to Solley et al., in which the most common ocular manifestation was a small area of retinal infiltrate, which occurred in 11 eyes $[1,2]$. In that series, neuroretinitis occurred in only 6 out of 37 eyes (16.2\%) [2].

The seroprevalence of antibodies to Bartonella henselae varies worldwide, ranging from $3.8 \%$ to $50 \%$ in various studies [26-28]. To the best of our knowledge, there is no available data on the seroprevalence of Bartonella henselae antibodies in our general population. Elevated immunoglobulin M (Ig M) or G (Ig G) for Bartonella henselae is suggestive of current or past infection [29]. However, the sensitivity of an Ig M assay using IFA has been found to be lower than that of enzyme immunoassay (EIA) Ig M (50\% IFA versus $71.4 \%$ EIA) in patients who fulfilled two or more criteria for catscratch disease [30]. Despite this, IFA remains the only test of bartonella infection available in our setting. Unfortunately, the IFA used in our bacteriology unit measures antibodies to both Bartonella henselae and Bartonella quintana; therefore, we were unable to differentiate between these Bartonella spp. infections. Polymerase chain reaction (PCR) is emerging as an alternative, highly sensitive method of identifying Bartonella spp. in ocular specimens [18, 21]. PCR hybridization assay has shown $86.4 \%$ sensitivity in patients who fulfill two or more criteria for cat-scratch disease and $100 \%$ sensitivity in patients who fulfill three or more criteria [27]. However, routine PCR is not readily done in our setting due to high cost. We foresee the need for better serological diagnostic tests to guide the diagnosis and treatment of bartonella infection in our practice.

Generally, cat-scratch disease is a self-limiting disease, with good visual prognosis, but treatment may hasten recovery $[20,31]$. Early antibiotic treatment may not only speed recovery, but also improve the final visual outcome [20]. According to Bass et al., the mean duration of illness was 2.8 weeks in those with treatment, compared to 14.5 weeks in those without treatment [32]. Rifampicin, gentamicin, cotrimoxazole, ciprofloxacin, and doxycycline have shown efficacy in the treatment of cat-scratch disease [20]. However, doxycycline is contraindicated in children and pregnant women. In mild to moderate disease, the use of oral azithromycin for five-day duration achieves more rapid resolution of lymphadenopathy than conservative management [33]. Azithromycin has become the preferred antimicrobial of choice in treating ocular bartonellosis in our setting due to issues with compliance (daily dosing) and its improved safety profile compared to doxycycline (which is given twice a day and tends to cause gastrointestinal upset). There is no prospective study on the efficacy of azithromycin in treating ocular bartonellosis. However, susceptibility evaluation of Bartonella henselae against macrolide antibiotics found that these antibiotics are useful in the treatment of cat-scratch disease [34].

The role of systemic corticosteroids in the treatment of cat-scratch disease is still controversial. Isolated case reports have demonstrated good response to corticosteroids, especially in cases where the presentation is atypical [35-38]. Only one study of ocular bartonellosis has discussed the role of corticosteroids, which gave a good outcome when used in combination with antibiotics, in a series of 14 patients in Japan [19]. Corticosteroids may help control the degree of intraocular inflammation and optic neuropathy due to 
cat-scratch disease. In our study, systemic corticosteroid therapy was used in patients who had significantly decreased visual acuity on presentation. However, we do not believe that topical corticosteroids play a significant role in the management of this condition, as their penetration to the posterior segment of the eye is limited.

Our study has several limitations. First, its retrospective nature limits the amount of information available. Secondly, although our data was collected from two institutions, the demographic and clinical features of this disease may show a wider global variation than currently reported, due to the different socioeconomic and bacteriology profile in different regions. Direct comparison with previous literature is also not possible due to the relatively small numbers of patients in most studies, as well as differences in study design, including inclusion criteria and type of serological assay used. Larger scale studies and standardized diagnostic criteria are required to consolidate our findings.

\section{Conclusion}

Ocular bartonellosis tends to present with blurring of vision, which is associated with prodromal symptoms in more than half of these cases. The most common ocular signs are neuroretinitis, papillitis, and uveitis. Azithromycin is a viable option for treatment. Systemic corticosteroids may be considered in those with poor visual acuity on presentation.

\section{Competing Interests}

The authors declare that there are no conflicts of interest in the publication of this paper.

\section{References}

[1] W. A. Solley, D. F. Martin, N. J. Newman et al., "Cat scratch disease: posterior segment manifestations," Ophthalmology, vol. 106, no. 8, pp. 1546-1553, 1999.

[2] A. L. L. Curi, D. MacHado, G. Heringer et al., "Cat-scratch disease: ocular manifestations and visual outcome," International Ophthalmology, vol. 30, no. 5, pp. 553-558, 2010.

[3] A. V. Gray, K. S. Michels, A. K. Lauer, and J. R. Samples, "Bartonella henselae infection associated with neuroretinitis, central retinal artery and vein occlusion, neovascular glaucoma, and severe vision loss," American Journal of Ophthalmology, vol. 137, no. 1, pp. 187-189, 2004.

[4] H. Martínez-Osorio, M. Calonge, J. Torres, and F. González, "Cat-scratch disease (ocular bartonellosis) presenting as bilateral recurrent iridocyclitis.," Clinical Infectious Diseases, vol. 40, no. 5, pp. e43-e45, 2005.

[5] A. Raihan, E. Zunaina, W. Wan-Hazabbah, H. Adil, and T. Lakana-Kumar, "Neuroretinitis in ocular bartonellosis: a case series," Clinical Ophthalmology, vol. 8, pp. 1459-1466, 2014.

[6] S. Rattanavong, P. E. Fournier, V. Chu et al., "Bartonella henselae endocarditis in Laos-'the unsought will go undetected,' PLoS Neglected Tropical Diseases, vol. 8, no. 12, article e3385, 2014.

[7] N. Sanchez Clemente, C. A. Ugarte-Gil, N. Solórzano et al., "Bartonella bacilliformis: a systematic review of the literature to guide the Research Agenda for Elimination," PLoS Neglected Tropical Diseases, vol. 6, no. 10, article e1819, 2012.
[8] A. R. Chaudhry, M. R. Chaudhry, J. C. Papadimitriou, and C. B. Drachenberg, "Bartonella henselae infection-associated vasculitis and crescentic glomerulonephritis leading to renal allograft loss," Transplant Infectious Disease, vol. 17, no. 3, pp. 411-417, 2015.

[9] G. Mirouse, A. Journe, L. Casabianca, P. E. Moreau, S. Pannier, and C. Glorion, "Bartonella henselae osteoarthritis of the upper cervical spine in a 14-year-old boy," Orthopaedics \& Traumatology: Surgery \& Research, vol. 101, no. 4, pp. 519-522, 2015.

[10] R. M. Chiuri, M. F. Matronola, C. Di Giulio, L. Comegna, F. Chiarelli, and A. Blasetti, "Bartonella henselae infection associated with autoimmune thyroiditis in a child," Hormone Research in Paediatrics, vol. 79, no. 3, pp. 185-188, 2013.

[11] J. A. Best and B. Price, "Central scotoma without prodromal illness caused by Bartonella henselae neuroretinitis," The American Journal of Emergency Medicine, vol. 31, no. 5, pp. 887.el887.e2, 2013.

[12] E. Brunetti, M. Fabbi, G. Ferraioli et al., "Cat-scratch disease in Northern Italy: atypical clinical manifestations in humans and prevalence of Bartonella infection in cats," European Journal of Clinical Microbiology and Infectious Diseases, vol. 32, no. 4, pp. 531-534, 2013.

[13] M. Eiger-Moscovich, R. Amer, M. Oray, K. F. Tabbara, I. TugalTutkun, and M. Kramer, "Retinal artery occlusion due to Bartonella henselae infection: a case series," Acta Ophthalmologica, vol. 94, no. 5, pp. e367-e370, 2016.

[14] A. Seth, U. Raina, S. Thirumalai, S. Batta, and B. Ghosh, "Fullthickness macular hole in Bartonella henselae neuroretinitis in an 11-year-old girl," Oman Journal of Ophthalmology, vol. 8, no. 1, pp. 44-46, 2015.

[15] D. H. Hamilton, K. M. Zangwill, J. L. Hadler, and M. L. Cartter, "Cat-scratch disease-connecticut, 1992-1993," Journal of Infectious Diseases, vol. 172, no. 2, pp. 570-573, 1995.

[16] M. G. Pennisi, F. Marsilio, K. Hartmann et al., "Bartonella Species Infection in Cats: ABCD guidelines on prevention and management," Journal of Feline Medicine and Surgery, vol. 15, no. 7, pp. 563-569, 2013.

[17] C. Ishida, H. Tsuneoka, H. Iino et al., "Bartonella henselae infection in domestic cat and dog fleas," Kansenshogaku Zasshi, vol. 75, no. 2, pp. 133-136, 2001.

[18] F. T. Kerkhoff, A. M. C. Bergmans, A. Van Der Zee, and A. Rothova, "Demonstration of Bartonella grahamii DNA in ocular fluids of a patient with neuroretinitis," Journal of Clinical Microbiology, vol. 37, no. 12, pp. 4034-4038, 1999.

[19] T. Kodama, H. Masuda, and A. Ohira, "Neuroretinitis associated with cat-scratch disease in Japanese patients," Acta Ophthalmologica Scandinavica, vol. 81, no. 6, pp. 653-657, 2003.

[20] J. B. Reed, D. K. Scales, M. T. Wong, C. P. Lattuada Jr., M. J. Dolan, and I. R. Schwab, "Bartonella henselae neuroretinitis in cat scratch disease: diagnosis, management, and sequelae," Ophthalmology, vol. 105, no. 3, pp. 459-466, 1998.

[21] P. P. Diniz, P. E. Velho, L. H. Pitassi et al., "Risk factors for Bartonella species infection in blood donors from Southeast Brazil," PLoS Neglected Tropical Diseases, vol. 10, no. 3, Article ID e0004509, 2016.

[22] E. T. Cunningham Jr. and J. E. Koehler, "Ocular bartonellosis," American Journal of Ophthalmology, vol. 130, no. 3, pp. 340-349, 2000.

[23] S. L. Chi, S. Stinnett, E. Eggenberger et al., "Clinical characteristics in 53 patients with cat scratch optic neuropathy," Ophthalmology, vol. 119, no. 1, pp. 183-187, 2012. 
[24] R. Ben-Ami, M. Ephros, B. Avidor et al., "Cat-scratch disease in elderly patients," Clinical Infectious Diseases, vol. 41, no. 7, pp. 969-974, 2005.

[25] T. A. Florin, T. E. Zaoutis, and L. B. Zaoutis, "Beyond cat scratch disease: widening spectrum of Bartonella henselae infection," Pediatrics, vol. 121, no. 5, pp. e1413-e1425, 2008.

[26] S. Bhengsri, H. C. Baggett, L. F. Peruski et al., "Bartonella seroprevalence in rural Thailand," Southeast Asian Journal of Tropical Medicine and Public Health, vol. 42, no. 3, pp. 687-692, 2011.

[27] H. Yoshida, N. Kusaba, K. Omachi et al., "Serological study of Bartonella henselae in cat scratch disease in Japan," Microbiology and Immunology, vol. 40, no. 9, pp. 671-673, 1996.

[28] A. Sander, M. Posselt, K. Oberle, and W. Bredt, "Seroprevalence of antibodies to Bartonella henselae in patients with cat scratch disease and in healthy controls: evaluation and comparison of two commercial serological tests," Clinical and Diagnostic Laboratory Immunology, vol. 5, no. 4, pp. 486-490, 1998.

[29] E. B. Suhler, A. K. Lauer, and J. T. Rosenbaum, "Prevalence of serologic evidence of cat scratch disease in patients with neuroretinitis," Ophthalmology, vol. 107, no. 5, pp. 871-876, 2000.

[30] A. M. C. Bergmans, M. F. Peeters, J. F. P. Schellekens et al., "Pitfalls and fallacies of cat scratch disease serology: evaluation of Bartonella henselae-based indirect fluorescence assay and enzyme-linked immunoassay," Journal of Clinical Microbiology, vol. 35, no. 8, pp. 1931-1937, 1997.

[31] J. Karolak and A. Gotz-Więckowska, "Neuroretinitis in cat scratch disease," Klinika Oczna, vol. 112, no. 4-6, pp. 131-134, 2010.

[32] A. M. Margileth, "Antibiotic therapy for cat-scratch disease: clinical study of therapeutic outcome in 268 patients and a review of the literature," Pediatric Infectious Disease Journal, vol. 11, no. 6, pp. 474-478, 1992.

[33] J. W. Bass, B. C. Freitas, A. D. Freitas et al., "Prospective randomized double blind placebo-controlled evaluation of azithromycin for treatment of cat-scratch disease," Pediatric Infectious Disease Journal, vol. 17, no. 6, pp. 447-452, 1998.

[34] T. J. Ives, P. Manzewitsch, R. L. Regnery, J. D. Butts, and M. Kebede, "In vitro susceptibilities of Bartonella henselae, B. quintana, B. elizabethae, Rickettsia rickettsii, R. conorii, R. akari, and R. prowazekii to Macrolide antibiotics as determined by immunofluorescent-antibody analysis of infected vero cell monolayers," Antimicrobial Agents and Chemotherapy, vol. 41, no. 3, pp. 578-582, 1997.

[35] K. Bryant and G. S. Marshall, "Hepatosplenic cat scratch disease treated with corticosteroids," Archives of Disease in Childhood, vol. 88, no. 4, pp. 345-346, 2003.

[36] E. M. Laswell, K. D. Chambers, D. R. Whitsel, and K. Poudel, "New-onset refractory status epilepticus in an adult with an atypical presentation of cat-scratch disease: successful treatment with high-dose corticosteroids," Pharmacotherapy, vol. 35, no. 6, pp. e106-e110, 2015.

[37] P. Lerdluedeeporn, P. Krogstad, R. L. Roberts, and E. R. Stiehm, "Oral se," Clinical Pediatrics, vol. 42, no. 1, pp. 71-73, 2003.

[38] K. D. Weston, T. Tran, K. N. Kimmel, and B. L. Maria, "Possible role of high-dose corticosteroids in the treatment of cat-scratch disease encephalopathy," Journal of Child Neurology, vol. 16, no. 10, pp. 762-763, 2001. 


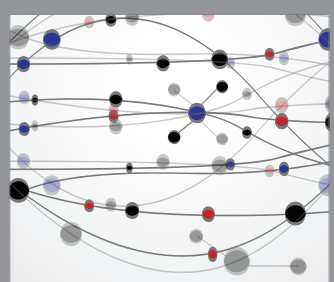

The Scientific World Journal
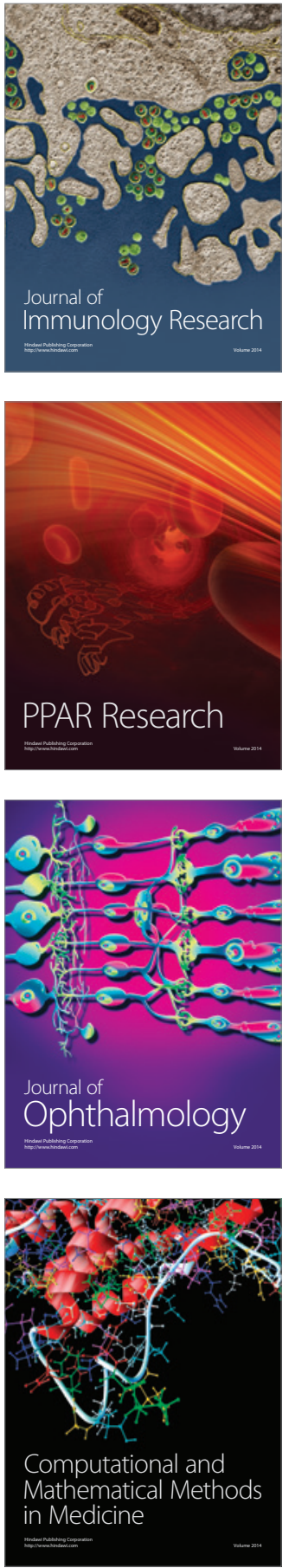

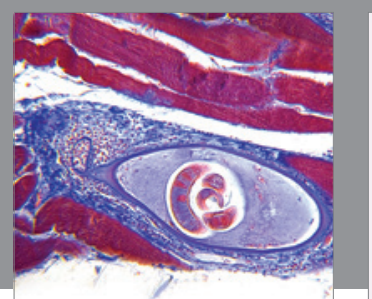

Gastroenterology Research and Practice
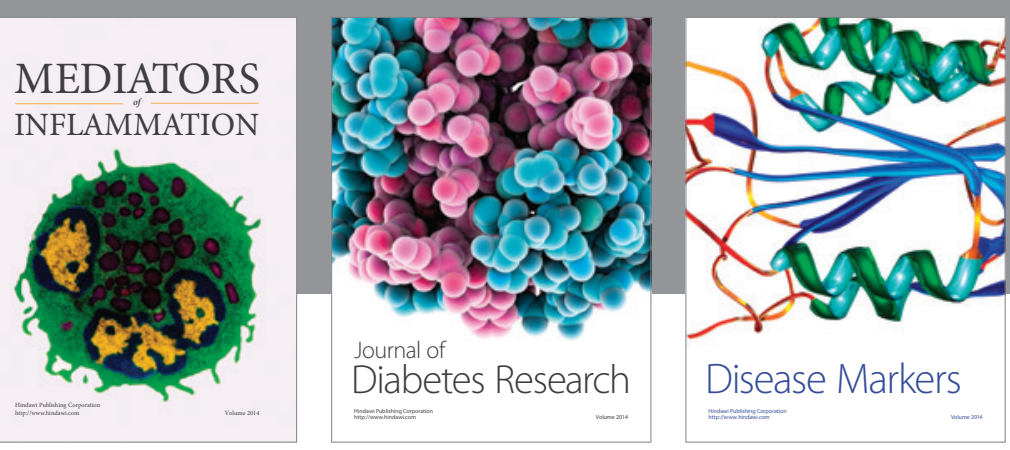

Disease Markers

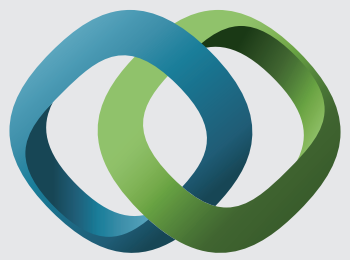

\section{Hindawi}

Submit your manuscripts at

https://www.hindawi.com
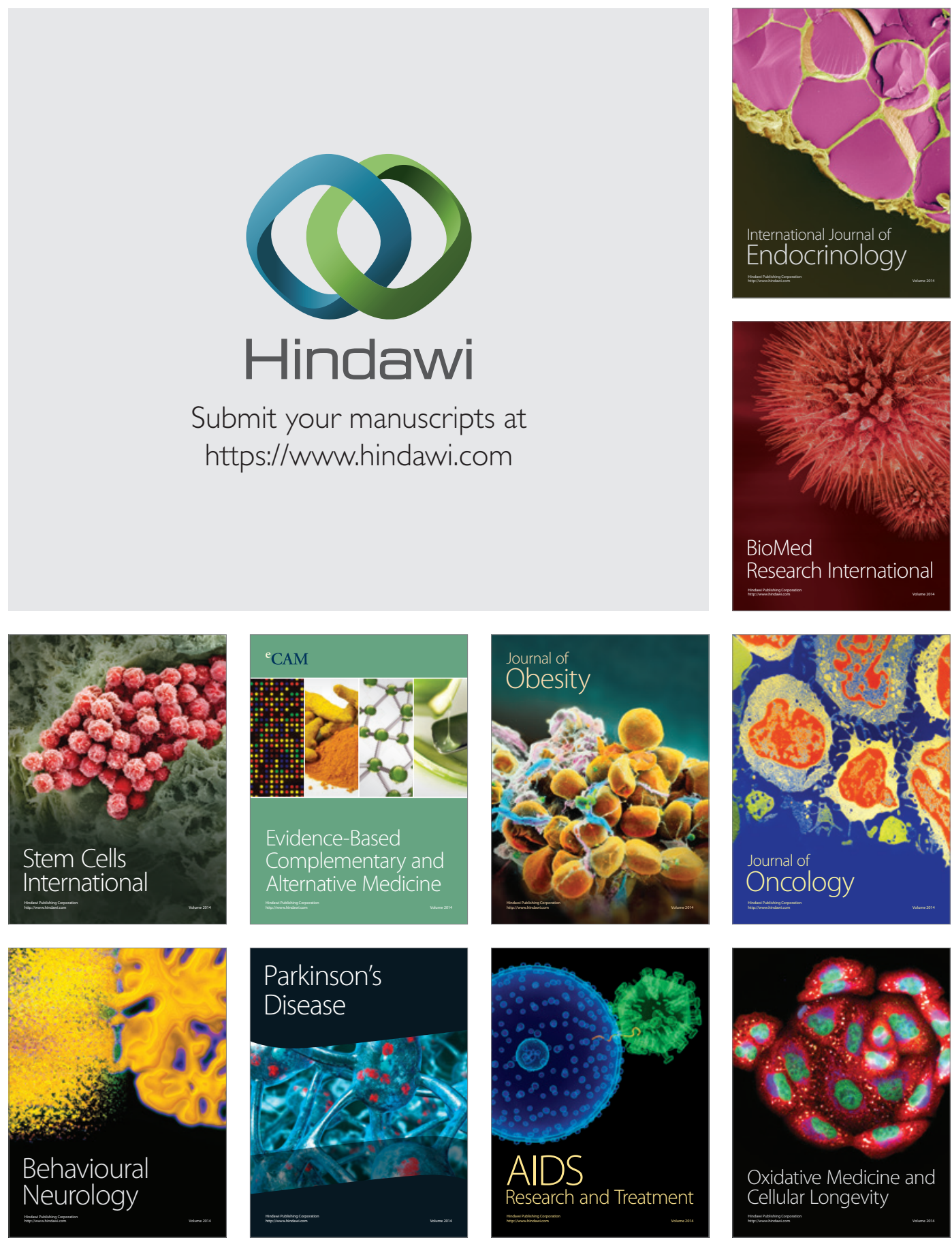